\title{
Effect of solvent, host precursor, dopant concentration and crystallite size on the fluorescence properties of $\mathrm{Eu}(\mathrm{III})$ doped yttria
}

\author{
Pramod K. Sharma * , R. Nass, H. Schmidt \\ Institut für Neue Materialien, Im Stadtwald, Geb. 43, 66123 Saarbrucken, Germany
}

\begin{abstract}
A systematic spectroscopic study of $\mathrm{Eu}^{3+}$ doped yttria powder phosphors are reported. Fluorescence study of this system has been investigated under the different solvent systems, e.g ethanol and water. In this study, the effect of the precursor of yttria, e.g. acetate, nitrate and chloride, on the fluorescence behaviour of $\mathrm{Eu}^{3+}$ have been observed in particular. Attention was paid to their effect on the emission intensity of the ${ }^{5} \mathrm{D}_{0} \rightarrow{ }^{7} \mathrm{~F}_{2}$ transition which is characteristic of the 'red fluorescence' of the $\mathrm{Eu}^{3+}$ ion. Results show that the $\mathrm{CH}_{3} \mathrm{COO}^{-}$ion has a tremendous effect on the enhancement of emission intensity, greater than $\mathrm{NO}_{3}^{-}$and $\mathrm{Cl}^{-}$ions. The Fourier transformed infrared (FTIR) spectroscopy was used for chemical and structural analysis. It has also been stated that the concentration quenching of dopant was more pronounced in the powders derived from yttrium chloride than from yttria precursor. In the case of $\mathrm{Y}\left(\mathrm{CH}_{3} \mathrm{COO}\right)_{3}$ as a precursor, the concentration quenching was totally absent and emission intensity increased with an increase in the concentration of dopant. This paper also includes some interesting results on the effect of modifiers, which are a mixture of Tween-80 with $\varepsilon$-caprolactum $/ \beta$ alanine/emulsogen-OG, on the ${ }^{5} \mathrm{D}_{0} \rightarrow{ }^{7} \mathrm{~F}_{2}$ transition of $\mathrm{Eu}^{3+}$ doped yttria in terms of crystallite size in brief.
\end{abstract}

Keywords Fluorescence; $\mathrm{Eu}_{2} \mathrm{O}_{3}, \mathrm{Y}_{2} \mathrm{O}_{3}$; Nanocrystallinity; Oxide

\section{Introduction}

Compounds containing rare earth have long been used as phosphors and laser materials because of their sharp, intensely luminescent $\mathrm{f}-\mathrm{f}$ electronic transitions [1,2]. In particular, europium(III) has five narrow emission bands corresponding to the ${ }^{5} \mathrm{D}_{0} \rightarrow{ }^{7}$ $\mathrm{F}_{i}$ transitions where $i=0,1,2,3$ and 4 . The strongest transition, ${ }^{5} \mathrm{D}_{0} \rightarrow{ }^{7} \mathrm{~F}_{2}$ occurs at approximately 610 $\mathrm{nm}$, which is characteristic of red fluorescence of

\footnotetext{
* Corresponding author. Fax: +496813025223.
}

$\mathrm{Eu}^{3+}$ [3]. This transition has also been shown to exhibit laser emission under appropriate conditions in $\mathrm{Eu}^{3+}$ doped crystals and glasses [4,5]. Significant research has been performed on the fluorescence properties of the $\mathrm{Eu}^{3+}$ ion in silica gel [6-8]. However, a detailed study of the incorporation of $\mathrm{Eu}^{3+}$ in yttria matrix is not well established. Since the optical and other sensitive fluorescence properties of doped yttria depend on the local structure and bonding of dopant cations, a detailed study of these factors is important from a device engineering perspective [9]. This work was undertaken in order to characterize 
the fluorescence behaviour of $\mathrm{Eu}^{3+}$ in the yttria matrix as a function of precursor salts, solvent, dopant concentration and modifiers, e.g. Tween- 80 with $\beta$ alanine, $\varepsilon$-caprolactum and emulsogen-OG.

\section{Experimental procedure}

A precursor solution was prepared by dissolving a known amount of yttria salt $(0.044 \mathrm{~mol})$ and $\mathrm{Eu}\left(\mathrm{NO}_{3}\right)_{3} 5 \mathrm{H}_{2} \mathrm{O}(3 \mathrm{~mol} \%)$ in solvent. The solution was stirred for $2 \mathrm{~h}$. The modifier solution was prepared by dissolving $10 \mathrm{wt} \%$ of surface modifier (1:1 mixture of Tween-80 and $\beta$-alanine or $\varepsilon$-caprolactum or emulsogen-OG) with respect to $\mathrm{Eu}_{2} \mathrm{O}_{3} / \mathrm{Y}_{2} \mathrm{O}_{3}$ in $50 \mathrm{ml}$ of aqueous ammonium hydroxide solution $(\mathrm{pH}>10)$ and stirred for $1 \mathrm{~h}$ at room temperature. The precursor solution was added to the modifier solution dropwise through a burette at a controlled rate (10-15 drops per min) with vigorous stirring which converted into gel. The gel was subjected to centrifugation at the rate of $6000 \mathrm{rpm}$ for $30 \mathrm{~min}$. The aqueous solution in the centrifugate was removed by refluxing in toluene using a water trap, then the toluene was removed by evaporation. The resulting powder was dried in the oven at $60^{\circ} \mathrm{C}$ for $24 \mathrm{~h}$. The final product was isolated as a white powder.

The infrared spectra of the samples were recorded on a Fourier-transformation infrared spectrometer (Brucker, IFS 25). The particle size was determined from the maxima of particle size distribution which was measured by dynamic light scattering (Malvern 4700). The crystalline phase was determined by powder X-ray diffraction (XRD) on D-500 Siemens powder diffractometer. The fluorescence spectra were measured with a Hitachi fluorescence spectrophotometer with a $150 \mathrm{~W}$ xenon lamp.

\section{Results and discussion}

\subsection{Effect of solvent and precursor salt}

It may be demonstrated that the ${ }^{5} \mathrm{D}_{0} \rightarrow{ }^{7} \mathrm{~F}_{2}$ and ${ }^{5} \mathrm{D}_{0} \rightarrow{ }^{7} \mathrm{~F}_{4}$ transition intensities are proportional to the Judd-Oflet $\left(\Omega^{2}\right.$ and $\left.\Omega^{4}\right)$ and intensity parameters $\left(\eta_{21}\right.$ and $\left.\eta_{41}\right)$, respectively [9]. The purely mag-
Table 1

Effect of solvent, precursor, modifier on the fluorescence intensity parameters $\left(\eta_{21}\right.$ and $\left.\eta_{41}\right)$ of $\mathrm{Eu}^{3+}$ doped yttria

\begin{tabular}{lllll}
\hline Precursor of yttria & Solvent & Modifier & $\eta_{21}$ & $\eta_{41}$ \\
\hline YN & EOH & TB & 1.85 & $0: 58$ \\
YN & W & TB & 1.51 & $0: 50$ \\
YCl & W & TB & 1.02 & $0: 35$ \\
YAc & W & TB & 1.55 & 0.51 \\
YN $^{a}$ & EOH & TC & 6.81 & 0.44 \\
YN $^{\text {a }}$ & EOH & TB & 7.14 & 0.46 \\
YN $^{\text {a }}$ & EOH & TE & 7.40 & 0.48 \\
\hline
\end{tabular}

$\mathrm{YN}: \mathrm{Y}\left(\mathrm{NO}_{3}\right)_{3} \cdot 5 \mathrm{H}_{2} \mathrm{O}, \mathrm{YAc}: \mathrm{Y}\left(\mathrm{CH}_{3} \mathrm{COO}\right)_{3} \cdot 4 \mathrm{H}_{2} \mathrm{O}, \mathrm{YCl}: \mathrm{YCl}_{3}$. $6 \mathrm{H}_{2} \mathrm{O}, \mathrm{W}$ : water, $\mathrm{EOH}$ : ethanol, TB: Tween- $80+\beta$-alanine; TC: Tween- $80+\varepsilon$-caprolactum; TE: Tween- $80+$ emulsogen-OG.

${ }^{\mathrm{a}}$ Powder after heat treatment at $450^{\circ} \mathrm{C}$.

netic dipolar ${ }^{5} \mathrm{D}_{0} \rightarrow{ }^{7} \mathrm{~F}_{1}$ transition can be taken as a reference, since its intensity is not sensitive to the ligand field. The relationships

$\eta_{21}={ }^{5} \mathrm{D}_{0} \rightarrow{ }^{7} \mathrm{~F}_{2} /{ }^{5} \mathrm{D}_{0} \rightarrow{ }^{7} \mathrm{~F}_{1}$

and

$\eta_{41}={ }^{5} \mathrm{D}_{0} \rightarrow{ }^{7} \mathrm{~F}_{4} /{ }^{5} \mathrm{D}_{0} \rightarrow{ }^{7} \mathrm{~F}_{1}$

have been used as a measure for $\Omega^{2}$ and $\Omega^{4}$ Judd-Oflet parameters [10]. Therefore, $\eta_{21}$ and $\eta_{41}$ were measured for various samples synthesized in different solvent and precursor salts of host material (refer to Table 1). Fig. 1 depicts the emission spectra of $\mathrm{Eu}^{3+}$ doped yttria synthesized in the presence of water and ethanol as a different solvent medium upon excitation of the ${ }^{5} \mathrm{D}_{0} \rightarrow{ }^{7} \mathrm{~F}_{2}$ transition at 394 $\mathrm{nm}$. The emission intensity of the ${ }^{5} \mathrm{D}_{0} \rightarrow{ }^{7} \mathrm{~F}_{2}$ peak $(610-620 \mathrm{~nm})$ in ethanol solvent was observed to be higher than that of water. The $\eta_{21}$ was also found to be sensitive to those variations. It can be assumed that the ${ }^{5} \mathrm{D}_{0}$ of the $\mathrm{Eu}^{3+}$ excited state was quenched by $\mathrm{OH}$ groups. These $\mathrm{OH}$ groups were found indeed in a large quantity in the aqueous medium as shown in the FTIR spectra in Fig. 2. The band due to the $\mathrm{M}-\mathrm{OH}$ stretching vibration and the presence of adsorbed water (3300-3400 and $\left.1640 \mathrm{~cm}^{-1}\right)$ decreased in intensity with the change in solvent from water to ethanol [11]. Brecher et al. demonstrated that the europium showed intense fluorescence in a variety of solutions and the emission cross section of these compounds was found to be superior in ethanol and methyl methacrylate [12]. 


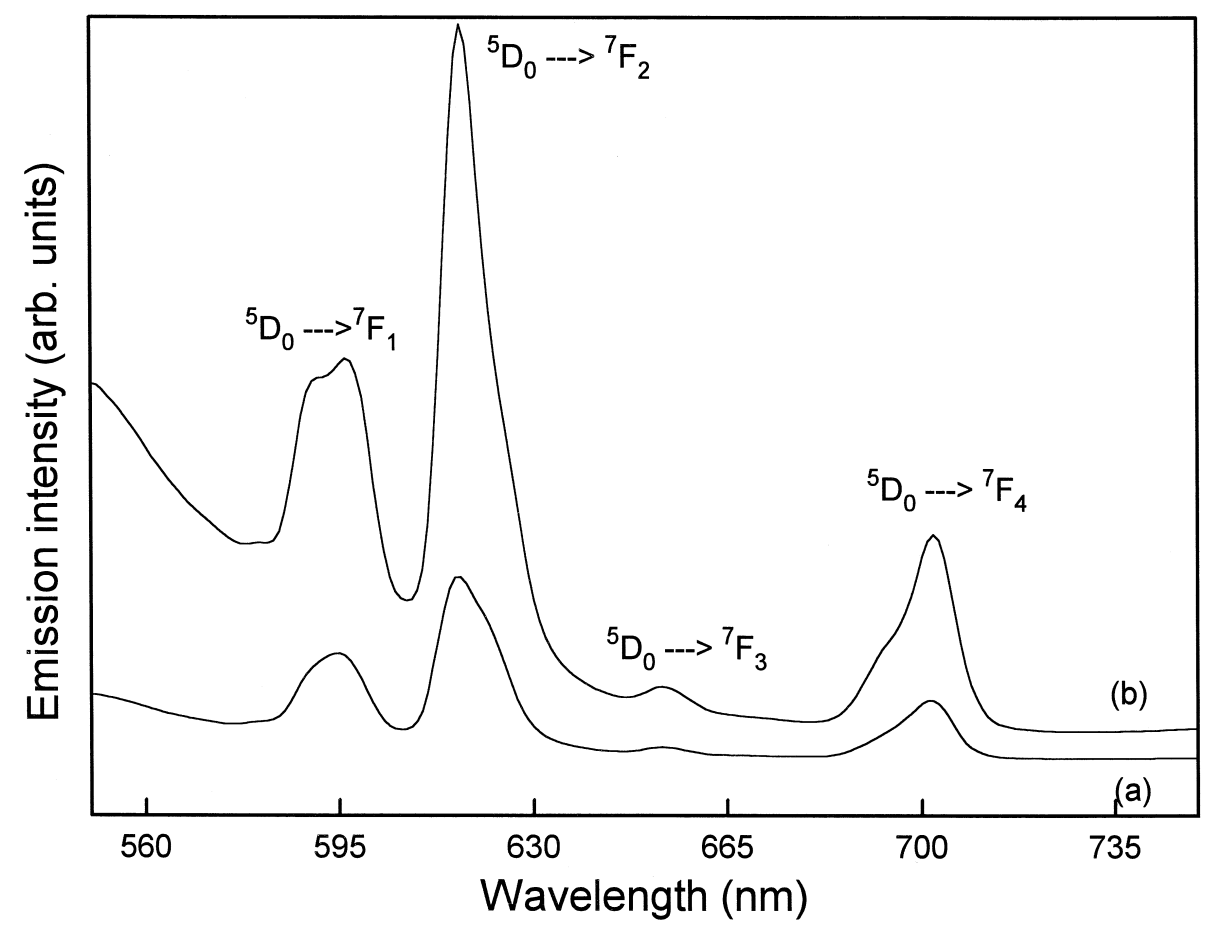

Fig. 1. Fluorescence spectra of $\mathrm{Eu}^{3+}$ doped yttria synthesized in solvent (a) water and (b) ethanol.

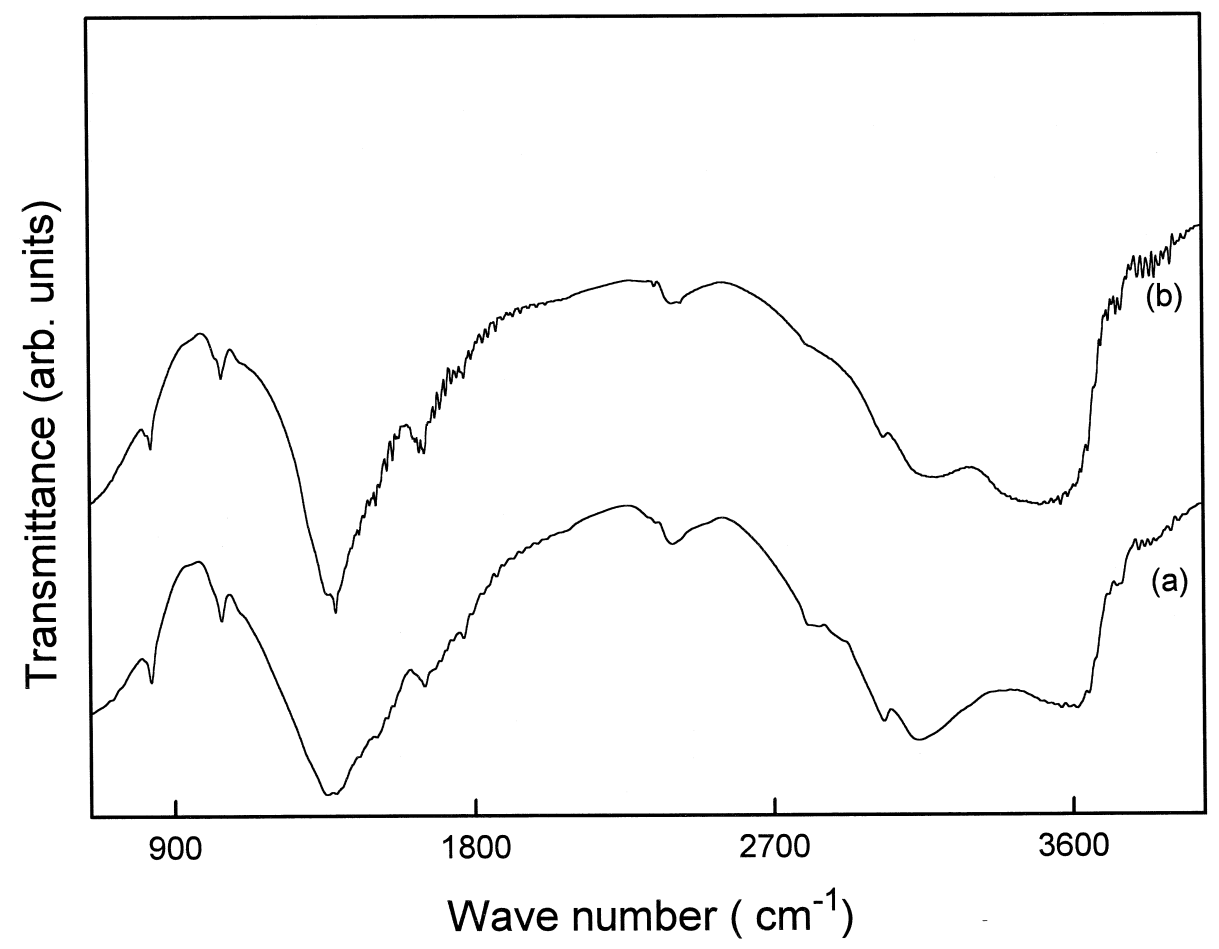

Fig. 2. FTIR spectra of $\mathrm{Eu}^{3+}$ doped yttria synthesized in (a) water and (b) ethanol medium. 


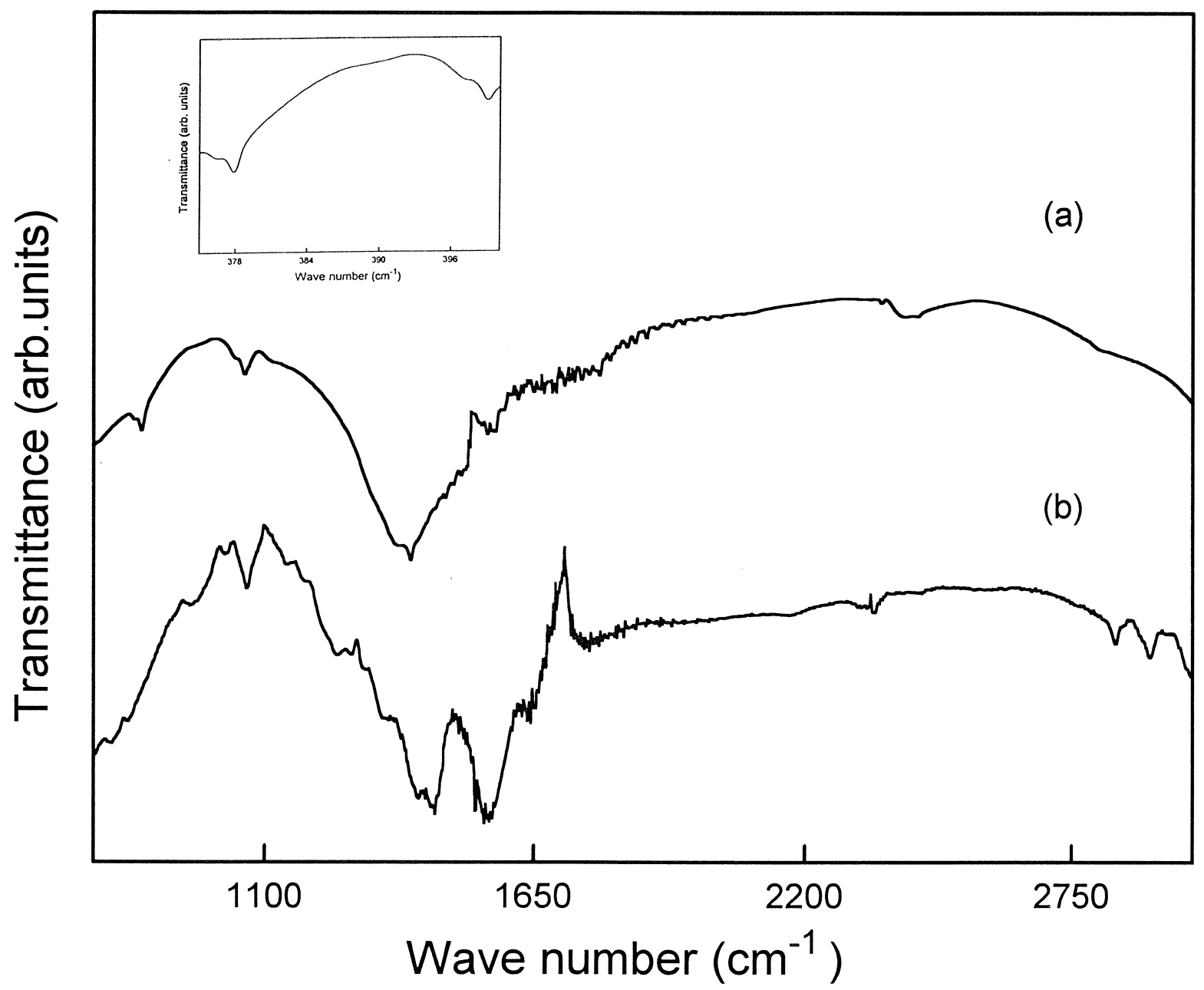

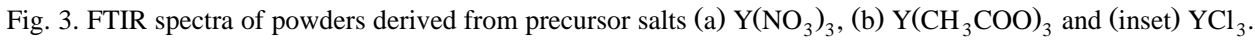

In ethanol, yttrium acetate has no solubility and yttrium chloride is sparingly soluble. Hence, water was chosen as the solvent medium to study the precursor effect of yttrium on the fluorescence properties of $\mathrm{Eu}^{3+}$ doped yttria. Fig. 3 presents the FTIR spectra of the powder derived from different precursors and indicates the presence of different anions associated with the powders. In Fig. 3(a) the peaks observed at 1545, 1337, 1030 and $820 \mathrm{~cm}^{-1}$ were believed to be due to nitrogen-oxygen stretching and bending [13]. Gotehouse et al. noted that the nitrate ions in the powder are supposed to exist in any of the three forms as shown in Fig. 4(a) [14]. Gotehouse and Curtis have explained that the separation of the two highest frequency bands is $\Delta \nu=120 \mathrm{~cm}^{-1}$ for the unidentate (St. II) nitrate group where as it is $\Delta \nu=190 \mathrm{~cm}^{-1}$ for the bidentate complex (St. III) $[14,15]$. As the separation of the two highest frequency bands from the FTIR spectra was found to be $\Delta \nu \approx 200 \mathrm{~cm}^{-1}$, it was suggested that the nitrate group coordinated with metal as a bidentate.

In this study the acetate anion may coordinate according to the modes shown in Fig. 4(b). The literature shows that the unidentate complex (St. V) exhibits $\Delta \nu$ values $\left(\nu_{\text {asym }}(\mathrm{COO})-\nu_{\text {sym }}(\mathrm{COO})\right)$ i.e. $\approx 435 \mathrm{~cm}^{-1}$ which are much greater than the ionic complex $\left(\approx 144 \mathrm{~cm}^{-1}\right)[16,17]$. The chelating (bidentate) complex (St. VI) exhibits $\Delta \nu$ values 
(a) Coordination of Nitrate anion with metal.
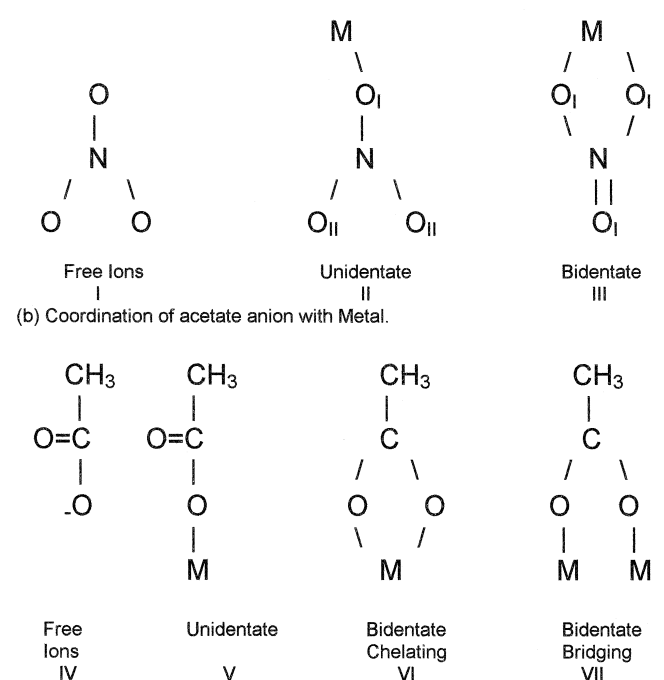

$$
\begin{gathered}
\text { Bidentate } \\
\text { Chelating } \\
\text { VI }
\end{gathered}
$$$$
\begin{aligned}
& \text { Bidentate } \\
& \text { Bridging } \\
& \text { VII }
\end{aligned}
$$

(C) Coordination of Chloride anion with Metal.

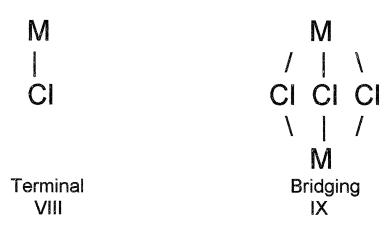

Fig. 4. Coordination of anion (a) nitrate, (b) acetate and (c) chloride with metal.

( $\approx 80 \mathrm{~cm}^{-1}$ ) which are significantly less than the ionic values. The $\Delta \nu$ value for the bridging complex (St. VII), i.e. $160 \mathrm{~cm}^{-1}$, are greater than those of the chelating complex and close to the ionic values [17]. The peak in Fig. 3(b) at $1355 \mathrm{~cm}^{-1}$ was assigned to be the stretching of $\mathrm{C}-\mathrm{C}$ bands. While the peaks at 1538 and $1410 \mathrm{~cm}^{-1}$ were due to stretching and bending of the $\mathrm{C}-\mathrm{O}$ bond, respectively [16]. The humps at around $2900 \mathrm{~cm}^{-1}$ were due to $\mathrm{C}-\mathrm{H}$ stretching [18]. A set of two bands appeared around 1723 and $1275 \mathrm{~cm}^{-1}$, which could be assigned to $\nu_{\text {asym }}(\mathrm{COO})$ and $\nu_{\text {sym }}(\mathrm{COO})$ vibrations of the acetate anion, respectively [16]. As $\Delta \nu$ was found to be 448 $\mathrm{cm}^{-1}$ it can, therefore, be assumed that the acetate ions are a ligand with a metal ion as unidentate $[19,20]$. The same FTIR spectra also shows peaks at 1545 and $1453 \mathrm{~cm}^{-1}$ with a frequency separation of $\Delta \nu=92 \mathrm{~cm}^{-1}$. Hence, it was suggested that the metal complex with acetate contains both types since it exhibit bands due to unidentate (1723 and 1275 $\mathrm{cm}^{-1}$ ) and bidentate (1545 and $1453 \mathrm{~cm}^{-1}$ ) with a frequency separation of $\Delta \nu=448 \mathrm{~cm}^{-1}$ and 92 $\mathrm{cm}^{-1}$, respectively.

Fig. 3 (inset) shows an FTIR spectra of the powder derived from chloride salt, clearly indicating a peak at $\approx 390 \mathrm{~cm}^{-1}$ which can be assigned for terminal coordination of chloride ions with metal since the terminal (St. VIII) and bridging (St. XI in Fig. 4(c)) bands appear between 400-200 $\mathrm{cm}^{-1}$ and $150-200 \mathrm{~cm}^{-1}$, respectively [20].

Fig. 5 shows the emission spectra of the powder derived from a different precursor. The peak locations in all the fluorescence spectra were essentially unperturbed, thus demonstrating the insensitivity of ${ }^{7} \mathrm{~F}$ transitions of the $\mathrm{Eu}^{3+}$ ion. The emission intensity of the ${ }^{7} F_{2}$ europium transition was found to be at least two-fold and five-fold more intense in the case of powder derived from yttrium acetate in comparison to the yttrium nitrate and chloride, respectively. These effects arise from an increased shielding of $\mathrm{Eu}^{3+}$ from quenching collisions in yttrium acetate and an increase in asymmetry in the $\mathrm{Eu}^{3+}$ site $[21,22]$. This results in an enhancement of the relative intensity of the different inorganic salts of europium (III) observed consistently in the previous work [11,12,22-24]. The ligand-ligand exchange phenomenon was presumed to play a role in this behaviour of $\mathrm{Eu}^{3+}$ which appears to activate the radiative mechanism in the case of yttrium acetate [21].

The trend for particle size, which was determined by dynamic light scattering for the powders derived from different concentrations of acetate salt at particular concentrations of modifier, was shown in Fig. 6. It was found to be almost constant with an increase in concentration of ions and this was obvious because size was controlled by the modifier during processing. However, the emission intensity of the ${ }^{5} \mathrm{D}_{0} \rightarrow{ }^{7} \mathrm{~F}_{2}$ transition was found to increase with the concentration of acetate ions as shown in Fig. 5. It can be explained in terms of the relative affinity of the counter ion for the inner coordination of the $\mathrm{Eu}^{3+}$ ion [7]. It has been found that acetate ions are more effective at entering the inner coordination sphere and result in less non-radiative decay, hence the emission intensity of the ${ }^{5} \mathrm{D}_{0} \rightarrow{ }^{7} \mathrm{~F}_{2}$ transition increased [7]. Therefore, it can be suggested that the anion has an influence on the fluorescence intensity 


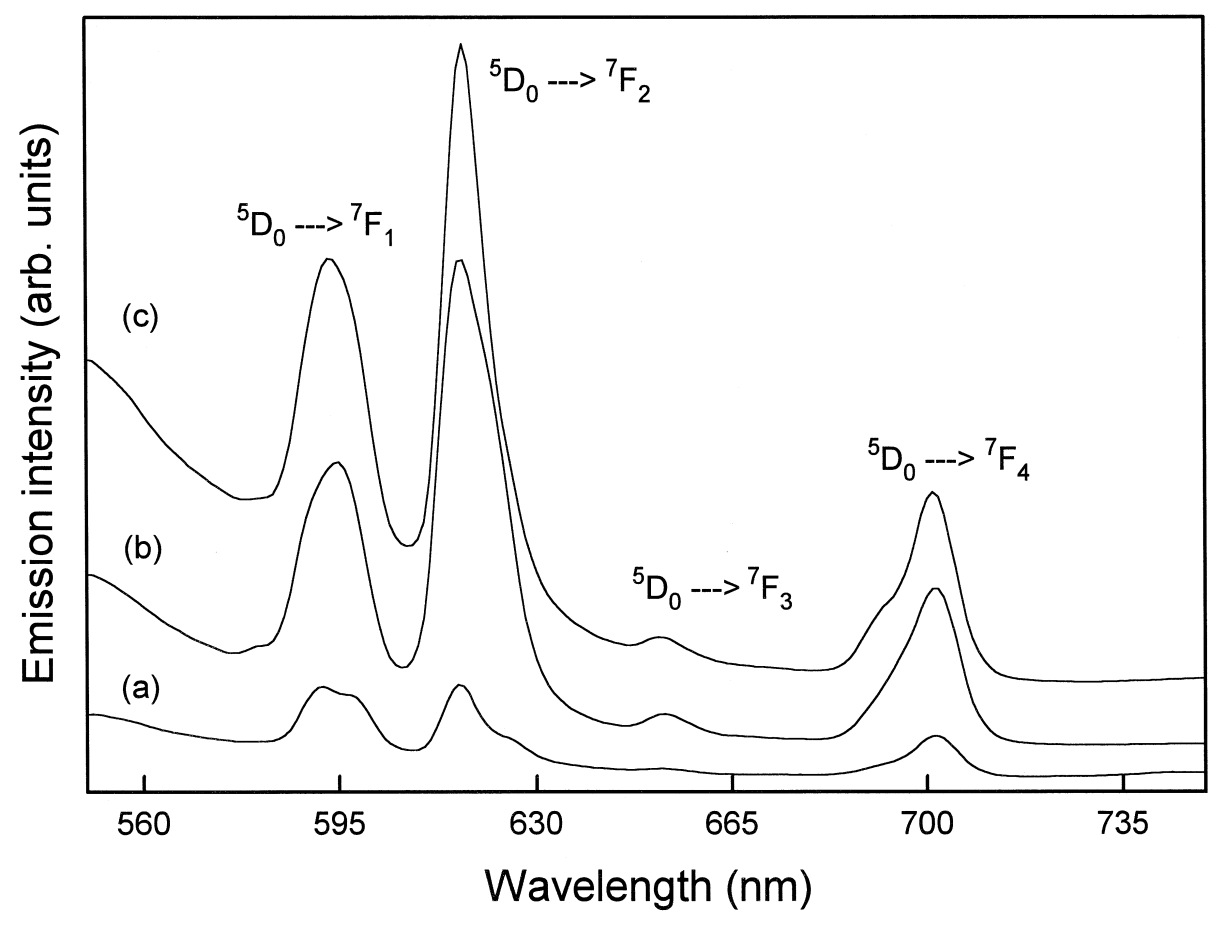

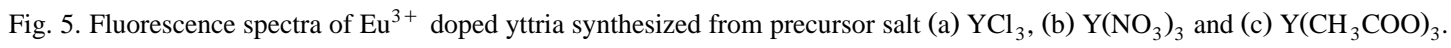

and it is in good agreement with earlier studies $[21,25]$.

\subsection{Effect of concentration of dopant}

Fig. 7 shows the emission intensity of $\mathrm{Eu}^{3+}$ ion in the yttrium acetate and yttrium chloride matrices
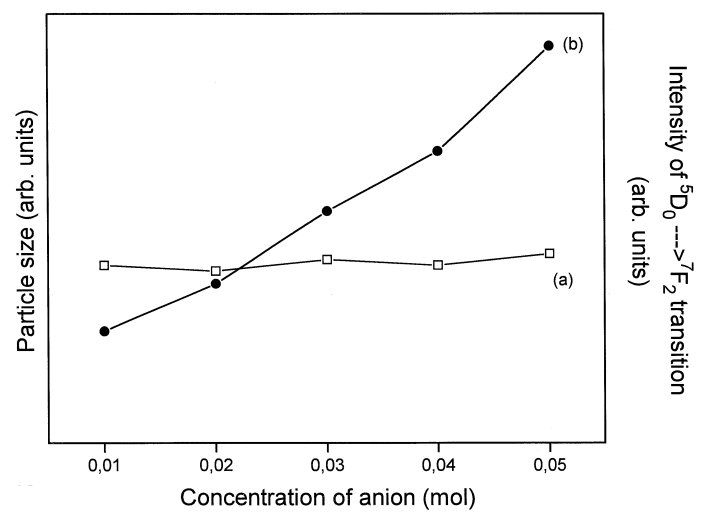

Fig. 6. Effect of concentration of anionic salt on (a) particle size and (b) ${ }^{5} \mathrm{D}_{0} \rightarrow{ }^{7} \mathrm{~F}_{2}$ transition. over 5 orders of dopant concentrations. The behaviour of $\mathrm{Eu}^{3+}$ was found to be highly concentration dependent in both the matrices. The emission intensity increased with an increase in the concentration of dopant in the case of yttrium acetate, indicated by sample radiances as a function of the concentration of dopant. In comparison, the emission

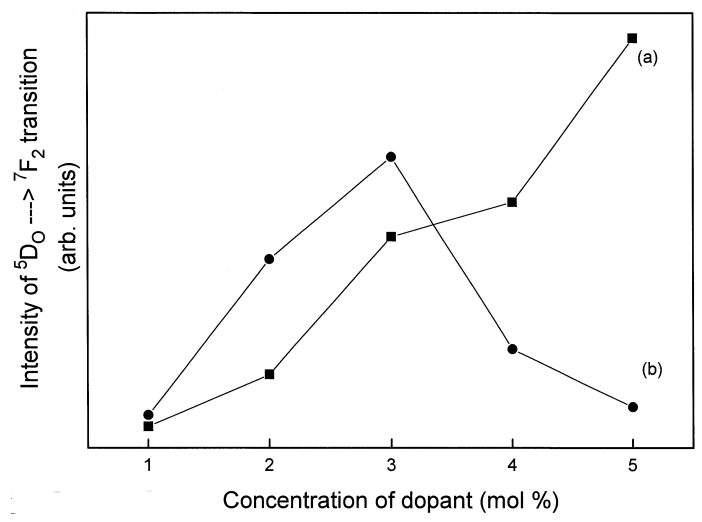

Fig. 7. Effect of concentration of $\mathrm{Eu}^{3+}(\mathrm{mol} \%)$ on emission intensity of ${ }^{5} \mathrm{D}_{0} \rightarrow{ }^{7} \mathrm{~F}_{2}$ transition in doped yttria derived from (a) $\mathrm{Y}\left(\mathrm{CH}_{3} \mathrm{COO}\right)_{3}$ and (b) $\mathrm{YCl}_{3}$. 
intensity increases to the peak maxima at a concentration of dopant upto $3 \mathrm{~mol} \%$ and then decreases with any increment in the case of yttrium chloride. This concentration quenching was caused by crossrelaxation between neighbouring $\mathrm{Eu}^{3+}$ ions. This was reduced somewhat in complexes (as the case of yttrium acetate) because interionic separations were increased relative to the uncomplexed ions [21]. In other words, the fluorescence intensity was proportional to the concentration of dopant in yttrium chloride until concentration quenching occurred.

\subsection{Effect of modifier on the fluorescence in terms of crystallite size}

In this work the effect of the modifier, e.g. Tween-80 with emulsogen-OG or $\varepsilon$-caprolactum or $\beta$-alanine, on the fluorescence properties of $\mathrm{Eu}^{3+}$ doped yttria was observed at a particular concentration of anionic salt in terms of crystallite size obtained after heat treatment at $450^{\circ} \mathrm{C}$. Burgard et al. [26] have demonstrated the effectiveness of surface modifier in controlling the nanocrystallite size by reducing the surface free energy of the particles to an appropriate level by the interaction of surface modifiers with generated particles for the $2 \mathrm{ZrO}_{2}-\mathrm{Y}_{2} \mathrm{O}_{3}$ system. The powder synthesized in the presence of a different modifier which is a mixture of Tween-80 with emulsogen-OG or $\beta$-alanine or $\varepsilon$-caprolactum, was found to have a crystallite size of the order of 12, 21 and $198 \mathrm{~nm}$, respectively. The crystallite size was determined from Scherrer's formula [27], i.e.

$B(2 \theta)=\frac{0.94 \lambda}{L \cos (\theta)}$

where $B(2 \theta)$ is the full width in radians subtended by the half maximum intensity width of the most intense peak in the X-ray diffraction pattern. The value of $\lambda$ is $1.54 \AA$. $L$ is the particle-size broadening, also supported by the particle size determination by the different modifiers, e.g. Tween- 80 with emulsogen-OG or $\varepsilon$-caprolactum or $\beta$-alanine, which was found to be 10, 23 and $181 \mathrm{~nm}$, respectively. Fig. 8

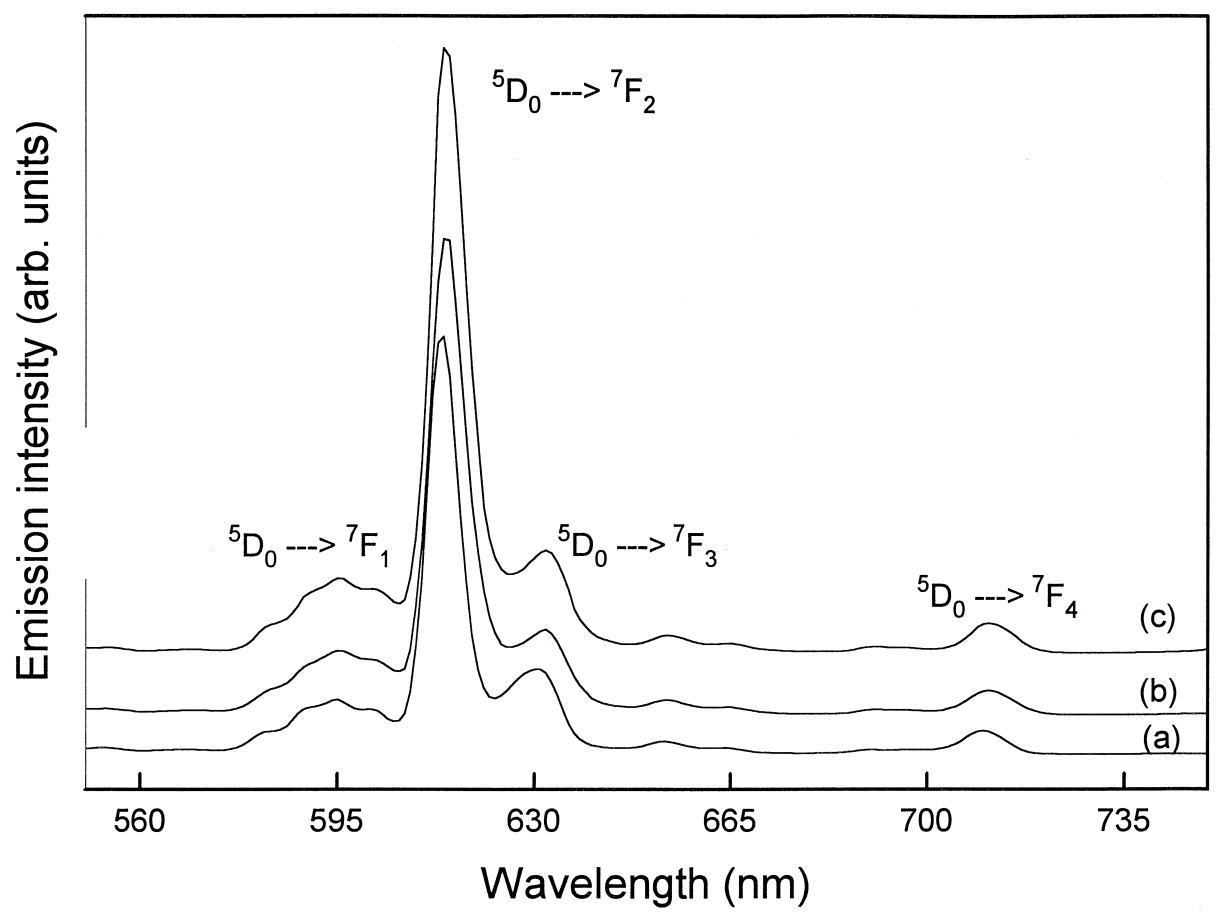

Fig. 8. Fluorescence spectra of $\mathrm{Eu}^{3+}$ doped yttria synthesized in presence of modifier (a) $\varepsilon$-caprolactum, (b) $\beta$-alanine and (c) emulsogen-OG. 


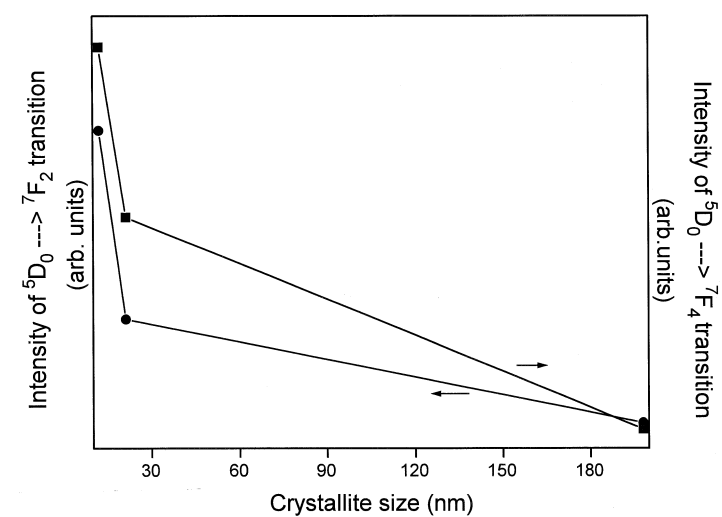

Fig. 9. Effect of crystallite size on the ${ }^{5} \mathrm{D}_{0} \rightarrow{ }^{7} \mathrm{~F}_{2}$ and ${ }^{5} \mathrm{D}_{0} \rightarrow{ }^{7} \mathrm{~F}_{4}$ transition of $\mathrm{Eu}^{3+}$ doped yttria.

shows a complete fluorescence spectra of $\mathrm{Eu}^{3+}$ doped yttria synthesized under the different modifiers. Fig. 9 depicts how the peak of emission intensity of the ${ }^{5} \mathrm{D}_{0} \rightarrow{ }^{7} \mathrm{~F}_{2}$ and ${ }^{7} \mathrm{~F}_{4}$ transitions increased as the crystallite size decreased. The size dependence of fluorescence can be explained in terms of the amount of particle unit area facing towards the incident light [28,29].

\section{Conclusions}

(1) The emission intensity was found to be higher in the case of ethanol than in water. This indicates that water has a strong affinity for providing a non-radiative decay path for excited $\mathrm{Eu}^{3+}$. Replacing water with ethanol results in a less effective nonradiative decay through $\mathrm{O}-\mathrm{H}$ oscillators and, hence, an increase in fluorescence intensity was observed.

(2) The precursor salts of the host have their own effect on the fluorescence intensity of $\mathrm{Eu}^{3+}$ ions. It has been found that the emission intensity decreases in the following order

\section{$\mathrm{CH}_{3} \mathrm{COO}^{-}>\mathrm{NO}_{3}^{-}>\mathrm{Cl}^{-}$}

The acetate ions were found to have a shielding effect on $\mathrm{Eu}^{3+}$, from quenching collisions in yttrium acetate, relative to chloride and nitrate. The $\mathrm{NO}_{3}^{-}$ ions have also shown an appreciable emission intensity, although the effect was found to be weaker than that observed in acetate ions. However, coordination of $\mathrm{Cl}^{-}$was terminal and $\mathrm{NO}_{3}^{-}$ions were assumed to be bidentate ligands while the acetate ion was assumed to coordinate as unidentate as well as bidentate with metal. On the other hand, the concentration of the anionic salt does not influence the particle size in the presence of a modifier but has an effect on the emission intensity of the ${ }^{7} \mathrm{~F}_{2}$ transition.

(3) The fluorescence intensity increases with the concentration of dopant in the case of yttrium acetate while concentration quenching was observed in $\mathrm{YCl}_{3}$.

(4) This study has shown that the modifier plays an important role in the enhancement of the fluorescence intensity of $\mathrm{Eu}^{3+}$ ion-doped yttria in terms of crystallite size. The crystallite size was found to decrease in the following order of modifiers:

$\varepsilon$-caprolactum $>\beta$-alanine $>$ emulsogen-OG,

while the order of emission intensity has been established as

$\varepsilon$-caprolactum $<\beta$-alanine $<$ emulsogen-OG.

\section{References}

[1] L.R. Mathews, E.T. Knobbe, Chem. Mater. 5 (1993) 1697.

[2] X.-P. Fan, M.-Q. Wang, G.-H. Xiong, J. Mater. Sci. Lett. 12 (1993) 1552.

[3] B.C. Joshi, J. Non-Cryst. Solids 180 (1995) 217.

[4] V.C. Costa, M.J. Lochhead, K.L. Bray, Chem. Mater. 8 (1996) 783.

[5] M.J. Waber, J. Non-Cryst. Solids 123 (1990) 208.

[6] K. Deviin, B.O. Kelly, Z.R. Tang, C. McDonagh, J.F. McGilp, J. Non-Cryst. Solids 135 (1991) 8.

[7] M.J. Lochhead, K.L. Bray, J. Non-Cryst. Solids 170 (1994) 143.

[8] C. Brecher, L.A. Riseberg, J. Non-Cryst. Solids 40 (1980) 469.

[9] S.J.L. Ribeiro, R.S. Hiratsuka, A.M.G. Massabni, M.R. Darolos, C.V. Santilli, J. Non-Cryst. Solids 147 (1992) 162.

[10] J. Huang, J. Loriers, P. Porcher, C.R. Acad. Sci. Paris 294 (1982) 545.

[11] X.-P. Fan, M.-Q. Wang, G.-H. Xiong, Mater. Sci. Eng. B 21 (1993) 55.

[12] C. Brecher, H. Samelson, A. Lempicki, J. Chem. Phys. 42 (1965) 1081.

[13] K. Richardson, M. Akinc, Cer. Inter. 13 (1997) 253.

[14] B.M. Gotehouse, S.E. Livinstone, S.C. Wallwork, R.S. Nyholm, J. Chem. Soc. 4222 (1957).

[15] N.F. Curtis, Y.M. Curtis, Inorg. Chem. 4 (1965) 804.

[16] J. Chalbi, M. Henry, H. Zarrouk, N. Gharbi, J. Livage, J. Non-Cryst. Solids 170 (1994) 1. 
[17] G.B. Deacon, R.J. Phillips, Coord. Chem. Rev. 33 (1980) 227.

[18] V. Jayaraman, T. Gnanasearan, G. Periaswami, Mater. Lett. 30 (1997) 157.

[19] S. Doeuf, M. Henry, C. Sanchez, J. Livage, J. Non-Cryst. Solids 89 (1987) 20.

[20] K. Nakamoto, Infrared and Raman Spectra of Inorganic and Coordination Compounds, 3rd ed., John Wiley, New York, 1978.

[21] L.R. Mathews, X.-J. Wang, E.-T. Knobbe, J. Non-Cryst. Solids 178 (1994) 44.

[22] L.R. Mathews, X.-J. Wang, E.-T. Knobbe, J. Sol-Gel, Sci. Technol. 2 (1992) 627.
[23] R. Camportrini, G. Carturan, M. Ferrari, M. Montagna, O. Pilla, J. Mater. Res. 7 (1992) 745.

[24] H. Mack, R. Reisfeld, D. Avnir, Chem. Phys. Lett. 99 (1983) 238.

[25] W. Grygiel, M. Starzok, J. Lumin. 71 (1997) 21.

[26] D. Burgard, C. Kropf, R. Nag, H. Schmidt, Better ceramics through chemistry, MRS 346 (1994) 101.

[27] R. Jagannathan, C. Athinarayanasamy, A. Mani, C.K. Jayasankar, Opt. Mater. 5 (1996) 57.

[28] S. Iwasaki, T. Ida, K. Kimura, Jpn. J. Appl. Phys. 35 (1996) L-551.

[29] P.K. Sharma, R. Naß, H. Schmidt, J. Lumin., communicated. 